\section{A TIGHT CONNECTION TO PRESERVE BONE}

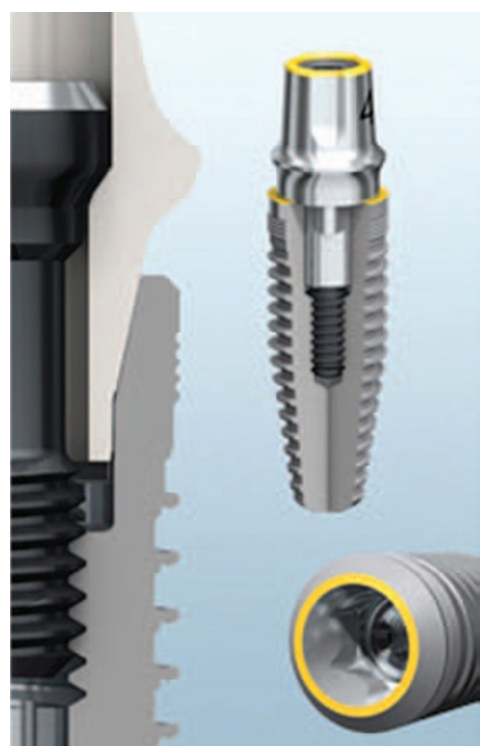

\section{SIR RAP'SHALLOT PROMOTES MOUTHWASH BRAND}

In October Listerine launched a new digital campaign, 'Mouth Off', aiming to raise awareness of all the things people put their mouths through.

The Listerine Mouth Off depicts the 'ultimate rap battle' that takes place between the mouth's worst enemies to determine who really is the 'baddest' for the mouth.

The six rappers battling it out for the title include some of the freshest UK rap talent such as Pen'alty - strong flow writer, weak gum biter; Miss Tea - the queen of stains; and Sir Rap'Shallot - a rap superstar with big beats but bad breath.

The host of the show, MC Total Care, announces the winner which is then followed by the ultimate 'beat-down rap', proving his seniority over any of the wannabe contenders because when it comes to mouths, nothing

is 'bad enough' to beat Listerine Total Care.

For the first time, Listerine also has a presence on Twitter, where the @ListerineUKMouthOff handle will allow fans to follow and interact with these outspoken chins.

To experience 'Mouth Off' for yourself, visit www.YouTube. com/ListerineUK.
Industry leader Nobel Biocare offers clinicians more restorative options than ever before with the new generation internal conical connection of the new NobelReplace Conical Connection implant.

Retaining the familiar tapered implant body to facilitate high primary stability, NobelReplace Conical Connection offers a safe and reliable solution for all indications in hard and soft bone. The implant introduces a tight conical connection to preserve marginal bone and an integrated platform shift to enhance soft tissue volume.

The versatile NobelReplace Conical Connection is an excellent implant system for experienced restorative dentists and newcomers alike, with the established step-bystep surgical protocol and colourcoded instruments.

Suitable for use with all prefabricated abutments and individualised CAD/CAM NobelProcera abutments, NobelReplace Conical Connection offers a tight seal and high mechanical strength, minimising the microgap and achieving natural looking aesthetics.

For more information, contact Nobel Biocare on 02087563300 , or visit www.nobelbiocare.com.

\title{
MINT, HONEYDEW OR STRAWBERRY PROPHYLAXIS
}

The new Flairesse prophylaxis system from DMG offers the choice between paste, foam, gel or varnish, all containing xylitol and fluoride.

Xylitol is an artificial sweetener which reduces the cariogenicity of plaque because it prevents the bacteria from adhering to the surfaces of the teeth as a biofilm.
Clinical studies have proven its cariostatic and anticariogenic effect.

With the Flairesse system, there is an option for each step: a prophylaxis paste to remove plaque and staining; gel or foam for fluoridation; and a varnish for desensitising exposed dentine tubules - all of which are free of added sugar and known allergens and taste pleasantly fresh. They are all available in mint and honeydew flavours, whilst the foam and gel are also available in strawberry.

For further information call 07854725544.

\section{PLACING A SPOTLIGHT ON CARIES}

The SIROInspect detection system developed by Sirona lights up caries so that dentists can reliably, quickly and simply identify where caries is concealed while excavating. The device is easy to operate and flexibly integrated into the workflow.

SIROInspect is based on Fluorescence-Aided Caries Excavation technology (FACE), which exploits the fluorescence characteristics of teeth. If teeth are illuminated with violet light in the spectrum of around $405 \mathrm{~nm}$, it doesn't only stimulate degradation products of caries bacteria but also healthy dentine to fluoresce. Healthy dental tissue lights up green whereas carious areas are visibly red. The triggers for the red colouring are porphyrin compounds which arise during the breakdown of hard dental substance by caries bacteria. The attending dentist can therefore see at first glance which areas are carious and which are not. The SIROInspect diagnostic glasses filter short wavelengths below $500 \mathrm{~nm}$. By contrast, light components with higher wavelengths remain visible.

The only thing users have to do during the treatment is to turn on the probe, put on the diagnostic glasses - and the check-up is ready to start.

\section{Contact Sirona}

Dental Systems on 08450715040 or emailinfo@ sironadental. co.uk.

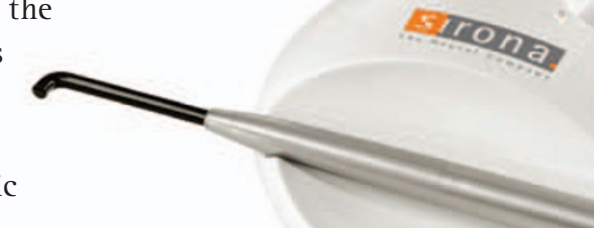

